K. Hayasida and H. Nagase

Nagoya Math. J.

Vol. 97 (1985), 51-69

\title{
ON SOLUTIONS OF VARIATIONAL INEQUALITIES CONSTRAINED ON A SUBSET OF POSITIVE CAPACITY
}

\author{
KAZUYA HAYASIDA AND HARUO NAGASE
}

1. Let $\Omega$ be a bounded domain of $R^{n}$ with boundary $\partial \Omega$ and let $E$ be a compact subset of $\Omega$. We assume that both $\partial \Omega$ and $E$ have positive capacity. The norm and the inner product in $L^{2}(\Omega)$ are simply denoted by \|\| and (, ) respectively. We define $\|u\|_{1}=\|\nabla u\|$. The completion of $C_{0}^{1}(\Omega)$ with respect to the norm $\|u\|_{1}$ is denoted by $H_{0}^{1}(\Omega)$, where $C_{0}^{1}(\Omega)$ is the set of all functions in $C^{1}(\Omega)$ with compact support in $\Omega$. The inner product of $H_{0}^{1}(\Omega)$ is written with $(,)_{1}$. We denote by $H^{-1}(\Omega)$ the dual space of $H_{0}^{1}(\Omega)$ and by \|\|$_{-1}$ its norm.

Let $K$ be a closed convex set in $H_{0}^{1}(\Omega)$ such that each element of $K$ is constraind only on $E$, that is, if $v \in H_{0}^{1}(\Omega)$ and $v=u$ on $E$ for some $u \in K$, then $v \in K^{1)}$. It is known that for any given $g \in H^{-1}(\Omega)$, there is a unique solution $u \in K$ of

$$
(u, v-u)_{1} \geqq(g, v-u) \quad \text { for all } v \in K
$$

and if $g$ is besides in $L_{\mathrm{loc}}^{2}(\Omega-E)$, the weak second derivatives $\partial^{2} u$ also are there, though $\partial^{2} u$ are distributions over $\Omega$.

In particular, when $g=0$ and $K$ equals to

$$
K_{1}=\left\{v \in H_{0}^{1}(\Omega) ; v \geqq \psi \text { on } E \text { in the sense of } H_{0}^{1}(\Omega)\right\}^{2)}
$$

for a given function $\psi \in C^{1}(\bar{\Omega}), \mathrm{H}$. Lewy and G. Stampacchia [11] showed that the solution $u$ of $(1.1)$ is in $C^{0}(\bar{\Omega})$ under certain assumptions on $E$ and $\partial \Omega$, for instance, $\Omega$ is a disk and $E$ is a segment. Their method is potential-theoretic.

Received July 6, 1983.

Revised October 17, 1983.

1) More precisely, there are two approximating sequences $\left\{u_{j}\right\},\left\{v_{j}\right\} \subset H_{0}^{1}(\Omega) \cap C^{0}(\Omega)$ such that $u_{j} \rightarrow u, v_{j} \rightarrow v$ in $H_{0}^{1}(\Omega)$ and $u_{j}=v_{j}$ on $E$. Thus $v=u$ on $E$ except for a set of capacity zero.

2) The precise definition of $K_{1}$ is referred to [11]. 
Our interest is to know the behavior of $\partial^{2} u$ in each neighborhood of $E$ and $\partial \Omega$. For this purpose we will give a result in the present paper (see Theorems 1 and 2 in this section), where it is stated for vector valued solutions, since there are several literatures for systems of variational inequalities (see e.g. [3]).

When $X$ is a Sobolev space defined on $\Omega,[X]^{N}$ denotes the space of $N$-vector valued functions with components in $X$. Let $U=\left(u_{1}, \cdots, u_{N}\right)$ and $V=\left(v_{1}, \cdots, v_{N}\right)$. Then we define

$$
\begin{aligned}
\|U\|^{2} & =\sum_{i=1}^{N}\left\|u_{i}\right\|^{2}, \quad(U, V)=\sum_{i=1}^{N}\left(u_{i}, v_{i}\right), \\
\|U\|_{1}^{2} & =\sum_{i=1}^{N}\left\|u_{i}\right\|_{1}^{2}, \quad(U, V)_{1}=\sum_{i=1}^{N}\left(u_{i}, v_{i}\right)_{1}
\end{aligned}
$$

and

$$
\|U\|_{-1}^{2}=\sum_{i=1}^{N}\left\|u_{i}\right\|_{-1}^{2}
$$

We consider the bilinear form

$$
a(U, V)=\left(a_{j k}^{(i)} \partial_{x_{j}} u_{i}, \partial_{x_{k}} v_{i}\right)+\left(b_{i j}^{(k)} \partial_{x_{k}} u_{j}, v_{i}\right)+\left(c_{i j} u_{j}, v_{i}\right),
$$

where the coefficients are all $C^{1}$-real valued in $\bar{\Omega}$ and the notation of each sum is abbreviated. We assume that for each $i,\left\{a_{j k}^{(i)}\right\}$ is symmetric and positive definite in $\bar{\Omega}$ and there is a positive constant $\alpha$ satisfying

$$
a(U, U) \geqq \alpha\|U\|_{1}^{2}
$$

for any $U \in\left[H_{0}^{1}(\Omega)\right]^{N}$. Obviously $a(U, V)$ is a continuous bilinear form on $\left[H_{0}^{1}(\Omega)\right]^{N}$.

Let $K$ be a closed convex set in $\left[H_{0}^{1}(\Omega)\right]^{N}$. For any given $G \in\left[H^{-1}(\Omega)\right]^{N}$, there is a unique solution $U \in K$ of

$$
a(U, V-U) \geqq(G, V-U) \quad \text { for all } V \in K
$$

(cf. Theorem 2.1, Chapter II in [9]). We assume that each element of $\boldsymbol{K}$ is constrained only on $E$ in the following sense: If $V \in\left[H_{0}^{1}(\Omega)\right]^{N}$ and $V=W$ on $E$ for some $W \in K$, then $V \in K$. We denote the coordinates of $R^{n}$ by $\left(x_{1}, \cdots, x_{n}\right)$. For example, $E$ lies on the hyperplane $\left\{x_{n}=0\right\}$ and

$$
\begin{aligned}
\boldsymbol{K} & =\boldsymbol{K}_{2} \\
& =\left\{V \in\left[H_{0}^{1}(\Omega)\right]^{N} ; \sum_{i=1}^{N}\left[\int_{E} v_{i}^{2} d x_{1} \cdots d x_{n-1}\right]^{1 / 2} \leqq 1\right\} .
\end{aligned}
$$


It is generally seen that the weak second derivatives $\partial^{2} U$ are in $\left[L_{\text {loc }}^{2}(\Omega\right.$ $-E)]^{N}$, if $G$ is there. Indeed $V+W \in K$, if $V \in K$ and $W \in\left[C_{0}^{1}(\Omega-E)\right]^{N}$.

We write $E \cup \partial \Omega$ simply with $F$. Let $\Phi(x)$ be the distance from the point $x$ to $F$. The set $F$ is said to satisfy $\left(C_{\delta}\right)$, if it holds that

$$
\int_{\Omega-F} \Phi(x)^{-\delta} d x<\infty
$$

We shall prove the following

TheOREM 1. Let $F$ satisfy $\left(C_{\hat{\delta}}\right)$ for a certain positive constant $\delta<1$ and let $\gamma=2 \delta /(2+\delta)$. If $\Phi G$ is in $\left[L^{2}(\Omega)\right]^{N}$, the solution $U$ of (1.3) satisfies

$$
\left[\int_{\Omega-F}\left|\partial^{2} U\right|^{r} d x\right]^{1 / r} \leqq C\left(\|\Phi G\|+\|G\|_{-1}+C(K)\right),
$$

where $C$ and $C(K)$ are independent of $G$ and $C$ does not depend on $K$.

The reason why we have imposed positive capacity on $\partial \Omega$ and $E$ is referred to Part I of [11].

Remark 1. If we do not impose (1.4) on $F$, it follows from the proof of Theorem 1 that

$$
\left\|\Phi \partial^{2} v\right\| \leqq \text { the right-hand side of (1.5) . }
$$

Remark 2. Theorem 1 holds also for quasi-linear systems of variational inequalities (see Section 5).

Next we consider the case when $E$ lies on a certain $(n-1)$-dimensional hyperplane, which is taken to be $\left\{x_{n}=0\right\}$ for simplicity. Let us denote by $\tilde{\partial} E$ the boundary of $E$ in $R^{n-1}$. In general, $\tilde{\partial} E$ is not equal to $\partial E$. For any $x \in R^{n}$ we denote by $\tilde{\Phi}(x)$ the distance from $x$ to $\tilde{\partial} E$. We shall say that $E$ satisfies $\left(D_{\delta^{\prime}}\right)$, if we have

$$
\int_{\Omega} \tilde{\Phi}(x)^{-\delta^{\prime}} d x<\infty
$$

Let $S$ be a given closed convex set in $R^{N}$. Let $K_{3}=\left\{V \in\left[H_{0}^{1}(\Omega)\right]^{N} ; V(x) \in S\right.$ for $x \in E$ except for a set of capacity zero $\}$, which is a special case of $K$ in Theorem 1 . Under these assumptions we shall prove also the following theorem for the solution $U$ of (1.3):

Theorem 2. Suppose that $K=K_{3}$. Let $E$ satisfy $\left(D_{\delta^{\prime}}\right)$ for a certain 
positive constant $\delta^{\prime}$ with $1 \leqq \delta^{\prime}<2$ and let $\gamma^{\prime}=2 \delta^{\prime} /\left(2+\delta^{\prime}\right)$. If $\tilde{\Phi} G$ is in $\left[L^{2}(\Omega)\right]^{N}$, we have for a neighborhood $B$ of $E$

$$
\left[\int_{B}\left|\partial^{2} U\right| r^{\prime} d x\right]^{1 / r^{\prime}} \leqq C\left(\|\tilde{\Phi} G\|+\|G\|_{-1}+C(K)\right),
$$

where $C$ and $C(K)$ are as described in Theorem 1. The integral domain in (1.7) is interpreted as $B-E$, because $\partial^{2} U$ is a distribution over $\Omega$.

In both theorems we note that $\gamma\left(\gamma^{\prime}\right)$ approaches $2 / 3(1)$, if $\delta\left(\delta^{\prime}\right)$ tends to $1(2)$, respectively. In the next section we shall give a few examples of sets satisfying $\left(C_{\delta}\right)$ or $\left(D_{\delta^{\prime}}\right)$, where $\delta\left(\delta^{\prime}\right)$ is taken as desired as close to $1(2)$.

We return to the variational inequalities (1.1) with a scalar unknown function. When $E$ is identical with $\Omega$ and $K$ is given in a concrete form such as $K_{1}$, there are many papers concerned with regularity of solutions as is well-known (see e.g. [2], [4] and [11]). J. Frehse [4] showed that $\partial^{2} u$ is in $L_{\text {loc }}^{\infty}(\Omega)$ under a certain assumption. When $E$ is a segment or an $(n-1)$-dimensional smooth manifold, the regularity of solutions for (1.1) was yielded by several authors (cf. e.g. [10], [1] and [5]). However, if $E$ is a proper subset of $\Omega$ and its figure is irregular, it seems to us that there are few literatures with respect to the regularity of solutions except for [11] and [15]. G. H. Williams [15] generalized the result of P. Hartman and G. Stampacchia [8]. On the other hand, if $\Omega$ is convex and $\partial \Omega$ is irregular, the unilateral problem imposed on $\partial \Omega$ was dealt by P. Grisvard [7], where he proved that $\partial^{2} u \in L^{2}(\Omega)$.

Our method is to use a parallel translation of test functions with a weight which do not change the set $F$. The simple translation parallel to the boundary is found in the book of J. L. Lions (see p. 256 in [12]), where $\Omega=\left\{x_{n}>0\right\}, K=K_{2}=\left\{v \in H^{1}(\Omega) ; v \geqq 0\right.$ on $\left.\partial \Omega\right\}$ and the square integrability of $\partial^{2} u$ is shown. He considered also the case when $\Omega$ is a cone (see Remark 1.17 in [13]). The line of our proof follows that of Theorem 8.6 in the above book.

The proofs of our theorems will be given in Section 4. For this we prepare several lemmas in Section 3. Section 5 is devoted to quasi-linear variational inequalities.

2. In this section we give two examples of compact sets with positive capacity, satisfying (1.4) or (1.6). We consider in $R^{2}$ with coordinates $(x, y)$. 
Example 1. Let $0<\delta<1$ and $0<b<a<1$. We define $Q=\{(x, y)$; $|x|<a,|y|<b\}$. By a proportional transformation we easily see that

$$
\iint_{Q}\left[\operatorname{dis}\left((x, y), Q^{c}\right)\right]^{-\delta} d x d y \leqq C(a b)^{1-\delta},
$$

where dis $\left((x, y), Q^{c}\right)$ is the distance from the point $(x, y)$ to $Q^{c}$ and the constant $C$ depends only on $\delta$.

Next we set

$$
\begin{aligned}
& A=\{|x|<a / 2,|y|<a / 2\} \cap[\{|x|<b / 2\} \cup\{|y|<b / 2\}], \\
& A_{1}=\{|x|<b / 2,|y|<b / 2\} \\
& A_{2}=\{|x|<b / 2, b / 2<y<a / 2\} \\
& A_{3}=\{|x|<b / 2,-a / 2<y<-b / 2\}, \\
& A_{4}=\{-a / 2<x<-b / 2,|y|<b / 2\}
\end{aligned}
$$

and

$$
A_{5}=\{b / 2<x<a / 2,|y|<b / 2\} .
$$

Then $A=\bigcup_{i=1}^{5} A_{i}$ and $\operatorname{dis}\left((x, y), A^{c}\right) \geqq \operatorname{dis}\left((x, y), A_{i}^{c}\right)$. Hence we see that

$$
\iint_{A}\left[\operatorname{dis}\left((x, y), A^{c}\right)\right]^{-\delta} d x d y \leqq \sum_{i=1}^{5} \iint_{A_{i}}\left[\operatorname{dis}\left((x, y), A_{i}^{c}\right)\right]^{-\delta} d x d y \text {. }
$$

Applying (2.1) to each integral on the right-hand side, we have

$$
\iint_{A}\left[\operatorname{dis}\left((x, y), A^{c}\right)\right]^{-\delta} d x d y \leqq C\left[b^{2(1-\delta)}+(b(a-b))^{1-\delta}\right] .
$$

Let $\left\{a_{j}\right\}$ be a monotone decreasing sequence such that

$$
\sum_{j=1}^{\infty} 4^{j} a_{j}^{2(1-\delta)}, \quad \sum_{j=1}^{\infty} 2^{j(1+\delta)} a_{j}^{1-\delta}<\infty
$$

and

$$
0<a_{j}<2^{1-j}\left(1-\sum_{i=1}^{\infty} 2^{i-1} a_{i}\right), \quad j=1,2, \cdots .
$$

We eliminate from [0, 1] an open interval $O_{1}^{(1)}$ with its length $a_{1}$ and with its center $1 / 2$. The remained closed intervals are denoted respectively by $I_{1}^{(1)}$ and $I_{2}^{(1)}$ in turn from the left. Secondly we eliminate from each $I_{j}^{(1)}$ an open interval $O_{j}^{(2)}$ with its length $a_{2}$ whose center is identical with that of $I_{j}^{(1)}$. Then there are remained four closed intervals, which are denoted by $I_{1}^{(2)}, I_{2}^{(2)}, I_{3}^{(2)}$ and $I_{4}^{(2)}$ in turn from the left, respectively. We 
repeat inductively this process, that is, $O_{j}^{(n+1)}$ is eliminated from the center of $I_{j}^{(n)}\left(j=1,2, \cdots, 2^{n}\right)$. The lastly remained closed set is written by $J$. And we set $E=J \times J$.

From (2.2) we have

$$
\begin{aligned}
& \iint_{\{[0,1] \times[0,1]\}-E}[\operatorname{dis}((x, y), E)]^{-\delta} d x d y \\
& \quad \leqq C\left[\sum_{j=1}^{\infty} 4^{j-1} a_{j}^{2(1-\delta)}+\sum_{j=1}^{\infty} 4^{j-1}\left(2^{1-j} a_{j}\left(1-\sum_{k=1}^{j} 2^{k-1} a_{k}\right)\right)^{1-\delta}\right] .
\end{aligned}
$$

By our hypothesis, the right-hand side is finite. This implies immediately that $E$ satisfies $\left(C_{\delta}\right)$. Since the one-dimensional measure of $J$ is positive, the capacity of $E$ is naturally so.

ExAmple 2. Let $1<\delta^{\prime}<2$ and $0<a \leqq 1$. Then we see that

$$
\int_{0}^{1} \int_{0}^{a}\left(x^{2}+y^{2}\right)^{-\delta^{\prime} / 2} d x d y \leqq C a^{2-\delta^{\prime}},
$$

where $C$ is independent of $a$.

Indeed, the left-hand side equals

$$
a^{2-\delta^{\prime}} \int_{0}^{1 / a} \int_{0}^{1}\left(s^{2}+t^{2}\right)^{-\delta^{\prime} / 2} d s d t .
$$

By putting $s=t s^{\prime}$, we see

$$
\begin{aligned}
& \int_{0}^{1 / a} \int_{0}^{1}\left(s^{2}+t^{2}\right)^{-\delta^{\prime} / 2} d s d t \\
& \quad=\left(\int_{0}^{1} \int_{0}^{1 / t}+\int_{1}^{1 / a} \int_{0}^{1 / t}\right) t^{1-\delta^{\prime}}\left(s^{\prime 2}+1\right)^{-\delta^{\prime} / 2} d s^{\prime} d t \\
& \quad \leqq \int_{0}^{1} t^{1-\delta^{\prime}} d t \cdot \int_{0}^{\infty}\left(s^{2}+1\right)^{-\delta^{\prime} / 2} d s^{\prime}+\int_{1}^{1 / a} t^{-\delta^{\prime}} d t
\end{aligned}
$$

which implies (2.3).

Let $\left\{I_{j}\right\}_{j=1}^{\infty}$ be a set of disjoint closed intervals contained in $[0,1]$. Setting $E=$ the closure of $\bigcup_{j=1}^{\infty} I_{j}$, we assume that the one-dimensional measure of $E-\bigcup_{j=1}^{\infty} I_{j}$ is zero. We put $(0,1)-E=\bigcup_{j=1}^{\infty} O_{j}$, where $\left\{O_{j}\right\}$ is a set of disjoint open intervals. Further we assume that $\sum_{j=1}^{\infty}\left|I_{j}\right|^{2-\delta^{\prime}}$ and $\sum_{j=1}^{\infty}\left|O_{j}\right|^{2-\delta^{\prime}}$ are both finite. Then from (2.3) it follows that

$$
\begin{aligned}
& \int_{-1}^{1} \int_{0}^{1} \tilde{\Phi}(x, y)^{-\delta^{\prime}} d x d y \\
& \quad=\sum_{j=1}^{\infty}\left(\int_{-1}^{1} \int_{I_{j}}+\int_{-1}^{1} \int_{O_{j}}\right) \tilde{\Phi}(x, y)^{-\delta^{\prime}} d x d y \\
& \quad \leqq C\left(\sum_{j=1}^{\infty}\left|I_{j}\right|^{2-\delta^{\prime}}+\sum_{j=1}^{\infty}\left|O_{j}\right|^{2-\delta}\right)<\infty .
\end{aligned}
$$


Hence $E$ satisfies $\left(D_{\delta^{\prime}}\right)$.

In this example Theorem 2 is available in the neighborhood of the set $E-\bigcup_{j=1}^{\infty} I_{j}$.

3. For the time being let $F$ be a general compact set in $R^{n}$. Let $\Phi(x)$ be the function as in the first section. We denote by $|x|$ the norm of the point $x$.

First we have

LEMMA 1. It holds that

$$
|\Phi(x)-\Phi(y)| \leqq|x-y| \quad \text { for } x, y \in R^{n}
$$

This lemma is well-known. So we omit the proof.

Let $h$ be a non-zero vector in $R^{n}$ with $h=|\boldsymbol{h}|$, where $h$ is assumed to be sufficiently small.

LEMma 2. We have for $x \notin F$

$$
(1+h)^{-1} \leqq \Phi(x) / \Phi(x+\Phi(x) h) \leqq(1-h)^{-1} \text {. }
$$

Proof. Let $z$ be a point in $F$ with $\Phi(x)=|x-z|$. We see

$$
\begin{aligned}
\Phi(x+\Phi(x) h) & \leqq|x+\Phi(x) h-z| \\
& \leqq|x-z|+h \Phi(x) .
\end{aligned}
$$

Thus we have shown the left half of the required inequalities.

Secondly let $z$ be a point in $F$ with $\Phi(x+\Phi(x) h)=|x+\Phi(x) h-z|$. Then we have

$$
\Phi(x) \leqq|x-z| \leqq|x+\Phi(x) h-z|+h \Phi(x),
$$

from which the lemma follows.

By Lemma 1 the function $\Phi$ is totally differentiable almost everywhere in $R^{n}$ and $\left|\partial_{x_{i}} \Phi\right| \leqq 1(i=1, \cdots, n)$. We consider the transformation of coordinates

$$
\Phi_{h}: y=x+\Phi(x) h
$$

We write $\boldsymbol{h}=\left(h_{1}, \cdots, h_{n}\right)$ and $\boldsymbol{J}=\partial\left(y_{1}, \cdots, y_{n}\right) / \partial\left(x_{1}, \cdots, x_{n}\right)$. Then we see

$$
J=\left[\begin{array}{c}
1+h_{1} \partial_{x_{1}} \Phi, h_{2} \partial_{x_{1}} \Phi, \cdots, h_{n} \partial_{x_{1}} \Phi \\
h_{1} \partial_{x_{2}} \Phi, 1+h_{2} \partial_{x_{2}} \Phi, \cdots, h_{n} \partial_{x_{2}} \Phi \\
\cdots \cdots \cdot \cdots, h_{n} \partial_{x_{n}} \Phi
\end{array}\right] .
$$


In virtue of Lemma 1 it follows that for $x, x^{\prime} \in R^{n}$

$$
\begin{aligned}
(1-h)\left|x-x^{\prime}\right| & \leqq\left|\Phi_{h}(x)-\Phi_{h}\left(x^{\prime}\right)\right| \\
& \leqq(1-h)^{-1}\left|x-x^{\prime}\right| .
\end{aligned}
$$

Accordingly, the mapping $\Phi_{h}$ and its inverse $\Phi_{h}^{-1}$ are both one-to-one mappings from $R^{n}$ onto itself. And they are totally differentiable almost everywhere in $R^{n}$, whose first derivatives are essentially bounded.

If we set $\Psi(y)=-\Phi(x)\left(=-\Phi\left(\Phi_{h}^{-1}(y)\right)\right)$, it is written

$$
\Phi_{h}^{-1}: x=y+\Psi(y) h .
$$

Now we define

$$
\begin{aligned}
& \left(S_{h} u\right)(x)=u(x+\Phi(x) h), \quad\left(T_{h} u\right)(y)=u(y+\Psi(y) h), \\
& \left(P_{h} u\right)(x)=h^{-1}\left[\left(S_{h} u\right)(x)-u(x)\right]
\end{aligned}
$$

and

$$
\left(Q_{h} u\right)(y)=h^{-1}\left[\left(T_{h} u\right)(y)-u(y)\right] .
$$

Obviously, $\left(Q_{h} u\right)(y)=-\left(P_{h} u\right)(x)$. These operators are similarly defined also for vector-valued functions.

The above definition of the quotient of differentials is originated from M. Marcus [14], where it was defined in a simple form:

$$
h^{-1}\left[u\left(x_{1}, \cdots, x_{i-1}, x_{i}+h x_{j}, x_{i+1}, \cdots, x_{n}\right)-u(x)\right]
$$

and it was used to the study of the Dirichlet problem with degenerate boundaries.

Hereafter, let the direction of $\boldsymbol{h}$ be arbitrarily fixed and let us put $\boldsymbol{e}=h^{-1} \boldsymbol{h}$. We write $\nabla_{x}=\left(\partial_{x_{1}}, \cdots, \partial_{x_{n}}\right)$ and $\nabla_{y}=\left(\partial_{y_{1}}, \cdots, \partial_{y_{n}}\right)$.

The following lemma is trivial. So we omit the proof.

LEMma 3. We have

$$
\begin{aligned}
& \nabla_{x}\left(S_{h} u\right)=S_{h} \nabla_{x} u+\left(h \cdot S_{h} \nabla_{x} u\right) \nabla_{x} \Phi, \\
& \nabla_{y}\left(T_{h} u\right)=T_{h} \nabla_{y} u+\left(h \cdot T_{h} \nabla_{y} u\right) \nabla_{y} \Psi, \\
& \nabla_{x}\left(P_{h} u\right)=P_{h} \nabla_{x} u+\left(e \cdot S_{h} \nabla_{x} u\right) \nabla_{x} \Phi
\end{aligned}
$$

and

$$
\nabla_{y}\left(Q_{h} u\right)=Q_{h} \nabla_{y} u+\left(e \cdot T_{h} \nabla_{y} u\right) \nabla_{y} \Psi .
$$

We note that the function $u$ in $H_{0}^{1}(\Omega)$ is extended over $R^{n}$ so that 
$u=0$ in $\Omega^{\mathrm{c}}$. We denoted by $(,)_{x}$ and \|\|$_{x}\left((,)_{y}\right.$ and \|\|$\left._{y}\right)$ the $L^{2}$-inner product and the $L^{2}$-norm of functions on $R_{x}^{n}\left(R_{y}^{n}\right)$, respectively. Let $J$ be the determinant of $J$, which is essentially positive in $R^{n}$.

Lemma 4. Let $u, v \in L^{2}\left(R^{n}\right)$. Then we have

$$
\left(Q_{h} u, v\right)_{y}=\left(J u, P_{h} v\right)_{x}+h^{-1}\left[(J u, v)_{x}-(u, v)_{x}\right] .
$$

If $u \in H_{0}^{1}(\Omega)$, there is a constant $C$ independent of $h$ and $u$ such that

$$
\left\|\Phi(x)^{-1} P_{h} u\right\|_{x} \leqq C\|u\|_{1}
$$

and

$$
\left\|\Psi(y)^{-1} Q_{h} u\right\|_{y} \leqq C\|u\|_{1} .
$$

Proof. The first equality is trivial. By the completion we can assume that $u \in C_{0}^{1}(\Omega)$. We see

$$
\begin{aligned}
\left(P_{h} u\right)(x) & =h^{-1} \int_{0}^{1} \partial_{\theta} u(x+\theta \Phi h) d \theta \\
& =\Phi \int_{0}^{1} e \cdot\left(\nabla_{x} u\right)(x+\theta \Phi h) d \theta .
\end{aligned}
$$

By Schwarz' inequality the first inequality is valid. The second inequality is similarly proved.

Q.E.D.

Lemma 5. Let $u$ be in $H_{0}^{1}(\Omega)$. Then

$$
\begin{aligned}
& \left\|P_{h} u-\Phi e \cdot \nabla_{x} u\right\|_{x}, \\
& \left\|Q_{h} u-\Psi e \cdot \nabla_{y} u\right\|_{y} \rightarrow 0 \quad(h \rightarrow 0) .
\end{aligned}
$$

Proof. If $u$ is in $C_{0}^{1}(\Omega)$, we have

$$
\begin{aligned}
& \left(P_{h} u\right)(x)-\Phi(x) e \cdot\left(\nabla_{x} u\right)(x) \\
& \quad=\Phi \int_{0}^{1} e \cdot\left[\left(\nabla_{x} u\right)(x+\theta \Phi h)-\left(\nabla_{x} u\right)(x)\right] d \theta .
\end{aligned}
$$

This means that

$$
\left\|P_{h} u-\Phi e \cdot \nabla_{x} u\right\|_{x}^{2} \leqq C \int_{0}^{1} \int\left|\left(\nabla_{x} u\right)(x+\theta \Phi h)-\left(\nabla_{x} u\right)(x)\right|^{2} d x d \theta,
$$

which is valid also for $u \in H_{0}^{1}(\Omega)$ by the completion. The right-hand side tends obviously to zero as $h \rightarrow 0$. The later half is quite similar. Q.E.D.

Now let $F$ be $E \cup \partial \Omega$ and $K$ be the closed convex set as in Theorem 1 . Then we have 
Lemma 6. If $U \in K, S_{h} U$ and $T_{h} U \in K$.

Proof. The function $S_{h} U$ is in $\left[H_{0}^{1}(\Omega)\right]^{N}$. Indeed, by Lemma 3 and the boundedness of $J^{-1}$ there is a constant $C$ such that

$$
\left\|S_{h} V\right\|_{1} \leqq C\|V\|_{1} \quad \text { for } V \in\left[C_{0}^{1}(\Omega)\right]^{N},
$$

where $C$ is independent of $h$ and $V$. And we note that the support of $S_{h} V$ is compact in $\Omega$, if $V$ is so. Thus taking an approximating sequence of $U$, we see that $S_{h} U \in\left[H_{0}^{1}(\Omega)\right]^{N}$. Since $S_{h} U=U$ on $E, S_{h} U \in K$ in view of the definition of $K$. Similarly $T_{h} U \in K$.

Q.E.D.

Next let $K_{3}$ be the set as in Theorem 2. Setting $F=\tilde{\partial} E \cup \partial \Omega$ and replacing $\boldsymbol{h}$ with $\boldsymbol{h}^{\prime}=\left(h_{1}, \cdots, h_{n-1}, 0\right)$, we consider $S_{\boldsymbol{h}^{\prime}}$ and $T_{h^{\prime}}$.

Then we have

Lemma $6^{\prime}$. If $U \in K_{3}, S_{h^{\prime}} U$ and $T_{h^{\prime}} U \in K_{3}$.

Proof. From the proof of the previous lemma, $S_{h^{\prime}} U$ and $T_{h^{\prime}} U$ are in $\left[H_{0}^{1}(\Omega)\right]^{N}$. We easily see that it is necessary and sufficient to be $x \in E$, in order that $x+h^{\prime} \Phi \in E$. And it is obvious that if $A$ is a set on $\left\{x_{n}=0\right\}$ with $(n-1)$-dimensional measure zero, the images $\Phi_{h^{\prime}}(A)$ and $\Phi_{h^{\prime}}^{-1}(A)$ have also measure zero on $\left\{x_{n}=0\right\}$. Thus $\left(S_{h^{\prime}} U\right)(x)$ and $\left(T_{h^{\prime}} U\right)(y) \in S$ for $x, y \in E$ except for a set of capacity zero, which completes the proof.

Q.E.D.

4. In this section we prove our theorems. First Theorem 1 will be shown.

Suppose that $U\left(=\left(u_{1}, \cdots, u_{N}\right)\right)$ is a solution of $(1.3)$ and $U^{(0)}$ is a particular solution of (1.3) for $G=(0, \cdots, 0)$. As well known, we have

$$
\left\|U-U^{(0)}\right\|_{1} \leqq \alpha^{-1}\|G\|_{-1} .
$$

that is,

$$
\|U\|_{1} \leqq \alpha^{-1}\|G\|_{-1}+C(K)
$$

(cf. Theorem 2.1, Chapter II in [9]).

Let us denote by $a(,)_{x}$ and $a(,)_{y}$ the bilinear form $a($,$) in Section$ 1 with respect to the variables $x$ and $y$, respectively.

We note that $S_{h} U$ and $T_{h} U \in K$ from Lemma 6 . We have by Lemma 3

$$
\begin{aligned}
a\left(U, S_{h} U\right)_{x}= & \left(a_{j k}^{(i)} \partial_{x_{j}} u_{i}, S_{h} \partial_{x_{k}} u_{i}\right)_{x}+\left(a_{j k}^{(i)} \partial_{x_{j}} u_{i},\left(h \cdot S_{h} \nabla_{x} u_{i}\right) \partial_{x_{k}} \Phi\right)_{x} \\
& +\left(b_{i j}^{(k)} \partial_{x_{k}} u_{j}, S_{h} u_{i}\right)_{x}+\left(c_{i j} u_{j}, S_{h} u_{i}\right)_{x} .
\end{aligned}
$$

In general, it holds that for any function $f, u$ and $v$ 


$$
\begin{aligned}
\left(f \partial_{x_{j}} u, S_{h} \partial_{x_{k}} v\right)_{x} & =\left(J^{-1} T_{h} f \cdot T_{h} \partial_{y_{j}} u, \partial_{y_{k}} v\right)_{y} \\
& =\left(J^{-1} T_{h} f \cdot \partial_{y_{j}} T_{h} u, \partial_{y_{k}} v\right)_{y}-\left(J^{-1} T_{h} f \cdot\left(h \cdot T_{h} \nabla_{y} u\right) \partial_{y_{j}} \Psi, \partial_{y_{k}} v\right)_{y} .
\end{aligned}
$$

Hence we see

$$
\begin{aligned}
a\left(U, S_{h} U\right)_{x}= & \left(J^{-1} T_{h} a_{j k}^{(i)} \cdot \partial_{y_{j}} T_{h} u_{i}, \partial_{y_{k}} u_{i}\right)_{y} \\
& +\left(J^{-1} T_{h} b_{i j}^{(k)} \cdot \partial_{y_{k}} T_{h} u_{j}, u_{i}\right)_{y}+\left(J^{-1} T_{h} c_{i j} \cdot T_{h} u_{j}, u_{i}\right)_{y} \\
& +\left(a_{j k}^{(i)} \partial_{x_{j}} u_{i},\left(\boldsymbol{h} \cdot S_{h} \nabla_{x} u_{i}\right) \partial_{x_{k}} \Phi\right)_{x} \\
& -\left(J^{-1} T_{h} a_{j k}^{(i)} \cdot\left(\boldsymbol{h} \cdot T_{h} \nabla_{y} u_{i}\right) \partial_{y_{j}} \Psi, \partial_{y_{k}} u_{i}\right)_{y} \\
& -\left(J^{-1} T_{h} b_{i j}^{(k)} \cdot\left(\boldsymbol{h} \cdot T_{h} \nabla_{y} u_{j}\right) \partial_{y_{k}} \Psi, u_{i}\right)_{y} .
\end{aligned}
$$

We put $J=1+h J_{1}$ and $J^{-1}=1+h J_{2}$. More precisely, $d y=J(x) d x$, $d x=J^{-1}(y) d y, J(x)=1+h J_{1}(x), J^{-1}(y)=1+h J_{2}(y)$ and $J(x) J^{-1}(y)=1$. It is easy to see that $J_{1}$ and $J_{2}$ are essentially bounded. We write $T_{h} f=f$ $+h Q_{h} f$. In this way, we arrive at the equality

$$
\begin{aligned}
a\left(T_{h} U, U\right)_{y}= & a\left(U, S_{h} U\right)_{x} \\
& -h\left[\left(\left(Q_{h}+J_{2}\right) a_{j k}^{(i)} \cdot \partial_{y_{j}} T_{h} u_{i}, \partial_{y_{k}} u_{i}\right)_{y}\right. \\
& \left.+\left(\left(Q_{h}+J_{2}\right) b_{i j}^{(k)} \cdot \partial_{y_{k}} T_{h} u_{j}, u_{i}\right)_{y}+\left(\left(Q_{h}+J_{2}\right) c_{i j} \cdot T_{h} u_{j}, u_{i}\right)_{y}\right] \\
& -h^{2}\left[\left(J_{2} Q_{h} a_{j k}^{(i)} \cdot \partial_{y_{j}} T_{h} u_{i}, \partial_{y_{k}} u_{i}\right)_{y}\right. \\
& \left.+\left(J_{2} Q_{h} b_{i j}^{(k)} \cdot \partial_{y_{k}} T_{h} u_{j}, u_{i}\right)_{y}+\left(J_{2} Q_{h} c_{i j} \cdot T_{h} u_{j}, u_{i}\right)_{y}\right] \\
& -\left(a_{j k}^{(i)} \partial_{x_{j}} u_{i},\left(h \cdot S_{h} \nabla_{x} u_{i}\right) \partial_{x_{k}} \Phi\right)_{x} \\
& +\left(T_{h} a_{j k}^{(i)} \cdot\left(h \cdot T_{h} \nabla_{y} u_{i}\right) \partial_{y_{j}} \Psi, \partial_{y_{k}} u_{i}\right)_{y} \\
& +h\left(J_{2} T_{h} a_{j k}^{(i)} \cdot\left(h \cdot T_{h} \nabla_{y} u_{i}\right) \partial_{y_{j}} \Psi, \partial_{y_{k}} u_{i}\right)_{y} \\
& +\left(T_{h} b_{i j}^{(k)} \cdot\left(h \cdot T_{h} \nabla_{y} u_{j}\right) \partial_{y_{k}} \Psi, u_{i}\right)_{y} \\
& +h\left(J_{2} T_{h} b_{i j}^{(k)} \cdot\left(h \cdot T_{h} \nabla_{y} u_{j}\right) \partial_{y_{k}} \Psi, u_{i}\right)_{y} .
\end{aligned}
$$

Moreover we see that

$$
\begin{aligned}
a\left(T_{h} U, T_{h} U\right)_{y}= & \left(a_{j k}^{(i)} T_{h} \partial_{y_{j}} u_{i}, T_{h} \partial_{y_{k}} u_{i}\right)_{y} \\
& +\left(b_{i j}^{(k)} T_{h} \partial_{y_{k}} u_{j}, T_{h} u_{i}\right)_{y}+\left(c_{i j} T_{h} u_{j}, T_{h} u_{i}\right)_{y} \\
& +\left(a_{j k}^{(i)} T_{h} \partial_{y_{j}} u_{i},\left(\boldsymbol{h} \cdot T_{h} \nabla_{y} u_{i}\right) \partial_{y_{k}} \Psi\right)_{y} \\
& \left.+a_{j k}^{(i)}\left(\boldsymbol{h} \cdot T_{h} \nabla_{y} u_{i}\right) \partial_{y_{j}} \Psi, T_{h} \partial_{y_{k}} u_{i}\right)_{y} \\
& +\left(a_{j k}^{(i)}\left(\boldsymbol{h} \cdot T_{h} \nabla_{y} u_{i}\right)^{2} \partial_{y_{j}} \Psi, \partial_{y_{k}} \Psi\right)_{y} \\
& +\left(b_{i j}^{(k)}\left(\boldsymbol{h} \cdot T_{h} \nabla_{y} u_{j}\right) \partial_{y_{k}} \Psi, T_{h} u_{i}\right)_{y}
\end{aligned}
$$

Similarly in deriving (4.2), we have

$$
\begin{aligned}
a\left(T_{h} U, T_{h} U\right)_{y}= & a(U, U)_{x} \\
& +h\left[\left(J_{1} a_{j k}^{(i)} \partial_{x_{j}} u_{i}, \partial_{x_{k}} u_{i}\right)_{x}+\left(J_{1} b_{i j}^{(k)} \partial_{x_{k}} u_{j}, u_{i}\right)_{x}\right.
\end{aligned}
$$




$$
\begin{aligned}
& \left.+\left(J_{1} c_{i j} u_{j}, u_{i}\right)_{x}\right]-h\left[\left(Q_{h} a_{j k}^{(i)} \cdot T_{h} \partial_{y_{j}} u_{i}, T_{h} \partial_{y_{k}} u_{i}\right)_{y}\right. \\
& \left.+\left(Q_{h} b_{i j}^{(k)} \cdot T_{h} \partial_{y_{k}} u_{j}, T_{h} u_{i}\right)_{y}+\left(Q_{h} c_{i j} \cdot T_{h} u_{j}, T_{h} u_{i}\right)_{y}\right] \\
& +\left(a_{j k}^{(i)} T_{h} \partial_{y_{j}} u_{i},\left(h \cdot T_{h} \nabla_{y} u_{i}\right) \partial_{y_{k}} \Psi\right)_{y} \\
& +\left(a_{j k}^{(i)}\left(h \cdot T_{h} \nabla_{y} u_{i}\right) \partial_{y_{j}} \Psi, T_{h} \partial_{y_{k}} u_{i}\right)_{y} \\
& +\left(a_{j k}^{(i)}\left(h \cdot T_{h} \nabla_{y} u_{i}\right)^{2} \partial_{y_{j}} \Psi, \partial_{y_{k}} \Psi\right)_{y} \\
& +\left(b_{i j}^{(k)}\left(h \cdot T_{h} \nabla_{y} u_{j}\right) \partial_{y_{k}} \Psi, T_{h} u_{i}\right)_{y} .
\end{aligned}
$$

Combining (4.2) and this equality, we obtain

$$
\begin{aligned}
& a\left(Q_{h} U, Q_{h} U\right)_{y}=h^{-2}\left[a\left(T_{h} U, T_{h} U\right)_{y}-a\left(T_{h} U, U\right)_{y}+a\left(U, U-T_{h} U\right)_{y}\right] \\
& =h^{-2}\left[a\left(U, U-S_{h} U\right)_{x}+a\left(U, U-T_{h} U\right)_{y}\right] \\
& +h^{-1}\left[\left(J_{1} a_{j k}^{(i)} \partial_{x_{j}} u_{i}, \partial_{x_{k}} u_{i}\right)_{x}+\left(J_{2} a_{j k}^{(i)} \partial_{y_{j}} T_{h} u_{i}, \partial_{y_{k}} u_{i}\right)_{y}\right] \\
& +h^{-1}\left[\left(J_{1} b_{i j}^{(k)} \partial_{x_{k}} u_{j}, u_{i}\right)_{x}+\left(J_{2} b_{i j}^{(k)} \partial_{y_{k}} T_{h} u_{j}, u_{i}\right)_{y}\right] \\
& +h^{-1}\left[\left(J_{1} c_{i j} u_{j}, u_{i}\right)_{x}+\left(J_{2} c_{i j} T_{h} u_{j}, u_{i}\right)_{y}\right] \\
& +h^{-1}\left[\left(Q_{h} a_{j k}^{(i)} \cdot \partial_{y_{j}} T_{h} u_{i}, \partial_{y_{k}} u_{i}\right)_{y}-\left(Q_{h} a_{j k}^{(i)} \cdot T_{h} \partial_{y_{j}} u_{i}, T_{h} \partial_{y_{k}} u_{i}\right)_{y}\right] \\
& +h^{-1}\left[\left(Q_{h} b_{i j}^{(k)} \cdot \partial_{y_{k}} T_{h} u_{j}, u_{i}\right)_{y}-\left(Q_{h} b_{i j}^{(k)} \cdot T_{h} \partial_{y_{k}} u_{j}, T_{h} u_{i}\right)_{y}\right] \\
& +h^{-1}\left[\left(Q_{h} c_{i j} \cdot T_{h} u_{j}, u_{i}\right)_{y}-\left(Q_{h} c_{i j} \cdot T_{h} u_{j}, T_{h} u_{i}\right)_{y}\right] \\
& +h^{-1}\left[-\left(J_{2} T_{h} a_{j k}^{(i)} \cdot\left(h \cdot T_{h} \nabla_{y} u_{i}\right) \partial_{y_{j}} \Psi, \partial_{y_{k}} u_{i}\right)_{y}\right. \\
& \text { - } \left.\left(J_{2} T_{h} b_{i j}^{(k)} \cdot\left(h \cdot T_{h} \nabla_{y} u_{j}\right) \partial_{y_{k}} \Psi, u_{i}\right)_{y}\right] \\
& +h^{-2}\left[\left(a_{j k}^{(i)}\left(h \cdot T_{h} \nabla_{y} u_{i}\right) \partial_{y_{j}} \Psi, T_{h} \partial_{y_{k}} u_{i}\right)_{y}\right. \\
& \left.+\left(a_{j k}^{(i)}\left(\boldsymbol{h} \cdot S_{h} \nabla_{x} u_{i}\right) \partial_{x_{j}} \Phi . \partial_{x_{k}} u_{i}\right)_{x}\right] \\
& +h^{-2}\left[a_{j k}^{(i)} T_{h} \partial_{y_{j}} u_{i},\left(\boldsymbol{h} \cdot T_{h} \nabla_{y} u_{i}\right) \partial_{y_{k}} \Psi\right)_{y} \\
& \text { - } \left.\left(T_{h} a_{j k}^{(i)} \cdot\left(\boldsymbol{h} \cdot T_{h} \nabla_{y} u_{i}\right) \partial_{y_{j}} \Psi, \partial_{y_{k}} u_{i}\right)_{y}\right] \\
& +h^{-2}\left[\left(b_{i j}^{(k)}\left(h \cdot T_{h} \nabla_{y} u_{j}\right) \partial_{y_{k}} \Psi, T_{h} u_{i}\right)_{y}\right. \\
& \text { - } \left.\left(T_{h} b_{i j}^{(k)} \cdot\left(h \cdot T_{h} \nabla_{y} u_{j}\right) \partial_{y_{k}} \Psi, u_{i}\right)_{y}\right] \\
& +\left[\left(J_{2} Q_{h} a_{j k}^{(i)} \cdot \partial_{y_{j}} T_{h} u_{i}, \partial_{y_{k}} u_{i}\right)_{y}+\left(J_{2} Q_{h} b_{i j}^{(k)} \cdot \partial_{y_{k}} T_{h} u_{j}, u_{i}\right)_{y}\right. \\
& \left.+\left(J_{2} Q_{h} c_{i j} \cdot T_{h} u_{j}, u_{i}\right)_{y}+\left(a_{j k}^{(i)}\left(e \cdot T_{h} \nabla_{y} u_{i}\right)^{2} \partial_{y_{j}} \Psi, \partial_{y_{k}} \Psi\right)_{y}\right] \text {. }
\end{aligned}
$$

Here we have used the fact that $\left\{a_{j k}^{(i)}\right\}$ is symmetric for each $i$. Denoting each bracket by $I_{j}$ on the right-hand side, we have

$$
a\left(Q_{h} U, Q_{h} U\right)_{y}=h^{-2} I_{1}+h^{-1} \sum_{j=2}^{8} I_{j}+h^{-2} \sum_{j=9}^{11} I_{j}+I_{12} .
$$

We shall prove the following inequality

$$
a\left(Q_{h} U, Q_{h} U\right)_{y} \leqq C\left(\|\Phi G\|+\|G\|_{-1}+\|U\|_{1}\right) \cdot\left(\|U\|_{1}+\left\|Q_{h} U\right\|_{1, y}\right),
$$

where $C$ is a constant independent of $h, U, G$ and $K$. For this sake let 
us estimate each term on the right-hand side of (4.3). From now on we denote simply by $C$ the constant independent of $h, U, G$ and $K$.

In virtue of (1.3) it follows that

$$
\begin{aligned}
a\left(U, U-S_{h} U\right)_{x} & \leqq\left(G, U-S_{h} U\right)_{x} \\
& =\left(T_{h} G, T_{h} U-U\right)_{y}+h\left(J_{2} T_{h} G, T_{h} U-U\right)_{y}
\end{aligned}
$$

and

$$
a\left(U, U-T_{h} U\right)_{y} \leqq\left(G, U-T_{h} U\right)_{y}
$$

Hence we obtain from Lemmas 2 and 4 that

$$
\begin{aligned}
h^{-2} I_{1} & \leqq\left|\left(Q_{h} G, Q_{h} U\right)_{y}\right|+\left|\left(J_{2} T_{h} G, Q_{h} U\right)_{y}\right| \\
& \leqq C\|\Phi G\|_{x}\left(\left\|Q_{h} U\right\|_{1, y}+\|U\|_{1}\right) .
\end{aligned}
$$

We use often Lemmas 3 and 4 without saying. Let us note that $J_{1}$ $+J_{2}=O(h)(h \rightarrow 0)$, more precisely, $J_{1}(x)+J_{2}(y)=O(h)(h \rightarrow 0)$, because $h^{-1}\left(J_{1}+J_{2}\right)=-J_{1} J_{2}$.

Secondly let us estimate $h^{-1} I_{2}$. Since

$$
\left(J_{2} a_{j k}^{(i)} T_{h} \partial_{y_{j}} u_{i}, \partial_{y_{k}} u_{i}\right)_{y}=\left(J_{J_{2}}(y) a_{j k}^{(i)}(y) \partial_{x_{j}} u_{i},\left(\partial_{x_{k}} u_{i}\right)(y)\right)_{x}
$$

we see

$$
\begin{aligned}
\left(J_{2} a_{j k}^{(i)} T_{h} \partial_{y_{j}} u_{i}, \partial_{y_{k}} u_{i}\right)_{y}-\left(J_{2}(y) a_{j k}^{(i)} \partial_{x_{j}} u_{i}, \partial_{x_{k}} u_{i}\right)_{x} \\
=\left((J-1) J_{2}(y) a_{j k}^{(i)}(y) \partial_{x_{j}} u_{i},\left(\partial_{x_{k}} u_{i}\right)(y)\right)_{x} \\
\quad+\left(\left(a_{j k}^{(i)}(y)-a_{j k}^{(i)}\right) J_{2}(y) \partial_{x_{j}} u_{i},\left(\partial_{x_{k}} u_{i}\right)(y)\right)_{x} \\
\quad+\left(J_{2}(y) a_{j k}^{(i)}\left(\left(\partial_{x_{k}} u_{i}\right)(y)-\partial_{x_{k}} u_{i}\right), \partial_{x_{j}} u_{i}\right)_{x}
\end{aligned}
$$

Hence

$$
\begin{aligned}
& h^{-1}\left|\left(J_{2} a_{j k}^{(i)} T_{h} \partial_{y_{j}} u_{i}, \partial_{y_{k}} u_{i}\right)_{y}-\left(J_{2}(y) a_{j k}^{(i)} \partial_{x_{j}} u_{i}, \partial_{x_{k}} u_{i}\right)_{x}\right| \\
& \quad \leqq C\left(\|U\|_{1}^{2}+\left\|P_{h} \nabla U\right\|_{x}\|U\|_{1}\right) \\
& \quad \leqq C\|U\|_{1}\left(\left\|Q_{h} U\right\|_{1, y}+\|U\|_{1}\right) .
\end{aligned}
$$

On the other hand

$$
\begin{aligned}
I_{2}= & \left(\left(J_{1}(x)+J_{2}(y)\right) a_{j k}^{(i)} \partial_{x_{j}} u_{i}, \partial_{x_{k}} u_{i}\right)_{x} \\
& +\left[\left(J_{2} a_{j k}^{(i)} T_{h} \partial_{y_{j}} u_{i}, \partial_{y_{k}} u_{i}\right)_{y}-\left(J_{2}(y) a_{j k}^{(i)} \partial_{x_{j}} u_{i}, \partial_{x_{k}} u_{i}\right)_{x}\right] \\
& +\left(J_{2} a_{j k}^{(i)}\left(h \cdot T_{h} \nabla_{y} u_{i}\right) \partial_{y_{j}} \Psi, \partial_{y_{k}} u_{i}\right)_{y} .
\end{aligned}
$$

This leads to the estimate

$$
h^{-1}\left|I_{j}\right| \leqq C\|U\|_{1}\left(\left\|Q_{h} U\right\|_{1, y}+\|U\|_{1}\right)
$$

for $j=2$. 
The term $I_{s}$ is written:

$$
\begin{aligned}
I_{5}= & -h\left(Q_{h} a_{j k}^{(i)} \cdot T_{h} \partial_{y_{j}} u_{i}, Q_{h} \partial_{y_{k}} u_{i}\right)_{y} \\
& +h\left(Q_{h} a_{j k}^{(i)} \cdot\left(e \cdot T_{h} \nabla_{y} u_{i}\right) \partial_{y_{j}} \Psi, \partial_{y_{k}} u_{i}\right)_{y} .
\end{aligned}
$$

Thus (4.5) is valid also for $j=5$. We see more easily that (4.5) holds for $j=3,4,6,7$ and 8 .

Let us now consider the term $I_{9}$. Obviously, $\nabla_{y} \Psi=-\left(\nabla_{x} \Phi\right)\left({ }^{t} J\right)^{-1}$. And we can write

$$
\left({ }^{t} \boldsymbol{J}\right)^{-1}=I+h H,
$$

where $I$ is the unit matrix and each component of $H$ is essentially bounded. Hence it is easily seen that

$$
\begin{aligned}
& \mid h^{-1}\left(h^{-1} I_{9}-\left[-\left(J a_{j k}^{(i)}(y)\left(e \cdot \nabla_{x} u_{i}\right) \partial_{x_{j}} \Phi, \partial_{x_{k}} u_{i}\right)_{x}+\left(a_{j k}^{(i)}\left(e \cdot S_{h} \nabla_{x} u_{i}\right) \partial_{x_{j}} \Phi, \partial_{x_{k}} u_{i}\right)_{x}\right] \mid\right. \\
& \quad \leqq C\|U\|_{1}^{2} .
\end{aligned}
$$

Using the equality

$$
\begin{aligned}
J a_{j k}^{(i)}(y) & \left(\boldsymbol{e} \cdot \nabla_{x} u_{i}\right)-a_{j k}^{(i)}\left(\boldsymbol{e} \cdot S_{h} \nabla_{x} u_{i}\right) \\
= & (J-1) a_{j k}^{(i)}(y)\left(e \cdot \nabla_{x} u_{i}\right)+\left(a_{j k}^{(i)}(y)-a_{j k}^{(i)}\right)\left(e \cdot \nabla_{x} u_{i}\right) \\
& +a_{j k}^{(i)}\left(\left(\boldsymbol{e} \cdot \nabla_{x} u_{i}\right)-\left(e \cdot S_{h} \nabla_{x} u_{i}\right)\right)
\end{aligned}
$$

we have

$$
\begin{aligned}
& h^{-1}\left|\left(J a_{j k}^{(i)}(y)\left(\boldsymbol{e} \cdot \nabla_{x} u_{i}\right) \partial_{x_{j}} \Phi, \partial_{x_{k}} u_{i}\right)_{x}-\left(a_{j k}^{(i)}\left(\boldsymbol{e} \cdot S_{\boldsymbol{h}} \nabla_{x} u_{i}\right) \partial_{x_{j}} \Phi, \partial_{x_{k}} u_{i}\right)_{x}\right| \\
& \quad \leqq C\|U\|_{1}\left(\left\|Q_{h} U\right\|_{1, y}+\|U\|_{1}\right) .
\end{aligned}
$$

Accordingly it follows that

$$
h^{-2}\left|I_{9}\right| \leqq C\|U\|_{1}\left(\left\|Q_{h} U\right\|_{1, y}+\|U\|_{1}\right) .
$$

More easily we can estimate $h^{-2}\left|I_{10}\right|, h^{-2}\left|I_{11}\right|$ and $\left|I_{12}\right|$ from above by the right-hand side of (4.5).

Therefore combining the above estimates and (4.3), we get (4.4). In virtue of (4.1) and (1.2), we obtain finally

$$
\left\|Q_{h} U\right\|_{1, y} \leqq C\left(\|\Phi G\|+\|G\|_{-1}+C(K)\right) .
$$

This implies that there are a subsequence $\left\{\boldsymbol{h}_{\nu}\right\}$ and a vector function $V \in$ $\left[H_{0}^{1}(\Omega)\right]^{N}$ such that $\left|\boldsymbol{h}_{\nu}\right| \rightarrow 0(\nu \rightarrow \infty)$ and $\left\{Q_{h_{\nu}} U\right\}$ converges weakly to $V$ in $\left[H_{0}^{1}(\Omega)\right]^{N}$ as $\nu \rightarrow \infty$. On the other hand, from Lemma $5\left\{Q_{h_{\nu}} U\right\}$ converges strongly to $\Psi e \cdot \nabla_{y} U$ in $\left[L^{2}(\Omega)\right]^{N}$ as $\nu \rightarrow \infty$. Thus $V=\Psi e \cdot \nabla_{y} U$. Writing $\Psi(y)=\Phi(y) \cdot(-\Phi(x) / \Phi(y))$, we have by Lemma 2 


$$
\left\|\Phi \boldsymbol{e} \cdot \nabla_{x} U\right\|_{1, x} \leqq C\left(\|\Phi G\|+\|G\|_{-1}+C(K)\right) .
$$

This yields

$$
\left\|\Phi \partial^{2} U\right\| \leqq C\left(\|\Phi G\|+\|G\|_{-1}+C(K)\right) .
$$

Let $\gamma$ and $\delta$ be the numbers as in Theorem 1. Let $p=\delta / \gamma$ and $q=$ 2/r. Then $p, q>1$ and $p^{-1}+q^{-1}=1$. Applying the Hölder's inequality, we conclude that

$$
\int_{\Omega-F}\left|\partial^{2} U\right|^{r} d x \leqq\left(\int_{\Omega-F} \Phi^{-\delta} d x\right)^{1 / p}\left(\int_{\Omega} \Phi^{2}\left|\partial^{2} U\right|^{2} d x\right)^{1 / q} .
$$

With the aide of (1.4) this completes the proof of Theorem 1. Q.E.D.

Next we shall prove Theorem 2.

Taking $S_{h^{\prime}}$ and $T_{h^{\prime}}$ as in Lemma $6^{\prime}$ and replacing $S_{h}\left(T_{h}\right)$ with $S_{h^{\prime}}\left(T_{h^{\prime}}\right)$ respectively, we proceed in parallel with the proof of Theorem 1 . In particular Lemma 6 is substituted for Lemma $6^{\prime}$. Then in place of (4.7) it follows that

$$
\left\|\Phi \boldsymbol{e}^{\prime} \cdot \nabla_{x} U\right\|_{1, x} \leqq C\left(\|\Phi G\|+\|G\|_{-1}+C\left(K_{3}\right)\right),
$$

where $\boldsymbol{e}^{\prime}=\boldsymbol{h}^{\prime}|| \boldsymbol{h}^{\prime} \mid$ and $\Phi(x)$ is the distance from $x$ to the set $\tilde{\partial} E \cup \partial \Omega$. Thus we get

$$
\left\|\Phi \partial \partial_{x_{j}} U\right\| \leqq C\left(\|\Phi G\|+\|G\|_{-1}+C\left(K_{3}\right)\right)
$$

for $j \neq n$.

Let $g_{i}$ be the $i$-th component of $G$. Since $-\partial_{x_{k}}\left(a_{j k}^{(i)} \cdot \partial_{x_{j}} u_{i}\right)+b_{i j}^{(k)} \partial_{x_{k}} u_{j}$ $+c_{i j} u_{j}=g_{i}$ in $\Omega-E$ for each $i$ and $a_{n n}^{(i)}$ does not vanish in $\bar{\Omega}$, (4.8) is valid also for $j=n$. Therefore

$$
\left(\int_{B} \Phi^{2}\left|\partial^{2} U\right|^{2} d x\right)^{1 / 2} \leqq \text { the right-hand side of (4.8). }
$$

In view of (1.6) the remained part is quite similar.

Q.E.D.

Remark. If $\|G\|_{-1}$ is small, Theorem 2 holds also for the set $K_{2}$ defined in the first section.

In fact, by putting $V=(0, \cdots, 0)$ in (1.3), we see that $\|U\|_{1} \leqq \alpha^{-1}\|G\|_{-1}$. Hence we have by the trace imbedding theorem

$$
\sum_{i=1}^{N}\left[\int_{E} u_{i}^{2} d x_{1} \cdots d x_{n-1}\right]^{1 / 2} \leqq \delta \quad(<1),
$$


which means that $S_{h^{\prime}} U$ and $T_{h^{\prime}} U$ are in $K_{2}$ for sufficiently small $\left|\boldsymbol{h}^{\prime}\right|$. Thus Theorem 2 holds for $K=K_{2}$.

5. In this section we remark that our theorems are valid also for quasi-linear systems of variational inequalities. For brevity we consider only single operators with simple form. In this direction there is a result of G. H. Williams [15], which is concerned with the Lipschitz regularity of the solutions in the case of $K=K_{1}$.

Let $a(\xi)=\left(a_{1}(\xi), \cdots, a_{n}(\xi)\right)$ be a $C^{1}$-mapping from $R^{n}$ to itself. We assume that there are two positive constants $\alpha$ and $M$ such that for all $\xi, \eta \in R^{n}$

$$
|a(\xi)| \leqq M(1+|\xi|)
$$

and

$$
\alpha|\xi|^{2} \leqq\left[\left(\nabla a_{i}\right)(\eta) \cdot \xi\right] \xi_{i} \leqq M|\xi|^{2} .
$$

The vector field $a(\xi)$ becomes strongly coercive monotone in the sense of Definition 4.1, Chapter III in [9]. We define

$$
a(u, v)=\left(a_{i}(\nabla u), \partial_{x_{i}} v\right) \quad \text { for } u, v \in H_{0}^{1}(\Omega) .
$$

Let $K$ be a closed convex set in $H_{0}^{1}(\Omega)$ and $g$ be any given function in $H^{-1}(\Omega)$. Then there exists a $u \in K$ of

$$
a(u, v-u) \geqq(g, v-u) \quad \text { for all } v \in K
$$

(cf. Theorem 1.4, Chapter III in [9]).

Let $u^{(0)}$ be a particular solution of (5.1) for $g=0$. We have by (5.1)

$$
\left(a_{i}(\nabla u), \partial_{x_{i}}\left(u^{(0)}-u\right)\right) \geqq\left(g, u^{(0)}-u\right)
$$

and

$$
\left(a_{i}\left(\nabla u^{(0)}\right), \partial_{x_{i}}\left(u-u^{(0)}\right)\right) \geqq 0 .
$$

Thus

$$
\left(a_{i}(\nabla u)-a_{i}\left(\nabla u^{(0)}\right), \partial_{x_{i}}\left(u-u^{(0)}\right)\right) \geqq\left(g, u-u^{(0)}\right) .
$$

The left-hand side equals

$$
\begin{gathered}
\int_{0}^{1}\left(\left(\nabla a_{i}\right)\left(t \nabla u+(1-t) \nabla u^{(0)}\right) \cdot \nabla\left(u-u^{(0)}\right), \partial_{x_{i}}\left(u-u^{(0)}\right)\right) d t \\
\geqq \alpha\left\|\nabla\left(u-u^{(0)}\right)\right\|^{2} \quad \text { (by our assumption) } .
\end{gathered}
$$

Hence 


$$
\left\|u-u^{(0)}\right\|_{1} \leqq \alpha^{-1}\|g\|,
$$

from which the following inequality holds in place of (4.1):

$$
\|u\|_{1} \leqq \alpha^{-1}\|g\|_{-1}+C(K) .
$$

In Theorem 1 we substitute $g$ and $u$ for $G$ and $U$ respectively. And we impose the assumptions of Theorem 1 . That is, $\Phi g$ is in $L^{2}(\Omega)$ and each element of $K$ is constrained only on $E$. Following the method of S. Gerhardt [6], we shall show that Theorem 1 holds for the solution $u$ of (5.1). S. Gerhardt [6] proved that $\partial^{2} u$ is in $L_{100}^{\circ}(\Omega)$ for the solution $u$ of (5.1) when $K=K_{1}$ and $\psi, g \in C^{2}(\Omega)$. Our proof is briefly sketched.

Setting

$$
I=a\left(u, u-S_{h} u\right)_{x}+a\left(u, u-T_{h} u\right)_{y},
$$

we have from (5.1)

$$
I \leqq\left(g, u-S_{h} u\right)_{x}+\left(g, u-T_{h} u\right)_{y},
$$

whence it follows that

$$
h^{-2} I \leqq C\|\Phi g\|_{x}\left(\left\|Q_{h} u\right\|_{1, y}+\|u\|_{1}\right) .
$$

Since

$$
\begin{aligned}
& a(u, u)_{x}=\left(J^{-1} a_{i}\left(T_{h} \nabla_{y} u\right), T_{h} \partial_{y_{i}} u\right)_{y}, \\
& a\left(u, S_{h} u\right)_{x}=\left(J^{-1} a_{i}\left(T_{h} \nabla_{y} u\right), \partial_{y_{i}} u\right)_{y}+\left(a_{i}\left(\nabla_{x} u\right),\left(h \cdot S_{h} \nabla_{x} u\right) \partial_{x_{i}} \Phi\right)_{x}
\end{aligned}
$$

and

$$
a\left(u, u-T_{h} u\right)_{y}=\left(a_{i}\left(\nabla_{y} u\right), \partial_{y_{i}} u-T_{h} \partial_{y_{t}} u\right)_{y}-\left(a_{i}\left(\nabla_{y} u\right),\left(h \cdot T_{h} \nabla_{y} u\right) \partial_{y_{i}} \Psi\right)_{y},
$$

we can write

$$
\begin{aligned}
I= & \left(a_{i}\left(T_{h} \nabla_{y} u\right)-a_{i}\left(\nabla_{y} u\right), T_{h} \partial_{y_{i}} u-\partial_{y_{i}} u\right)_{y} \\
& +h\left[\left(J_{2} a_{i}\left(T_{h} \nabla_{y} u\right), T_{h} \partial_{y_{i}} u-\partial_{y_{i}} u\right)_{y}-\left(a_{i}\left(\nabla_{x} u\right),\left(e \cdot S_{h} \nabla_{x} u\right) \partial_{x_{i}} \Phi\right)_{x}\right. \\
& \left.-\left(a_{i}\left(\nabla_{y} u\right),\left(e \cdot T_{h} \nabla_{y} u\right) \partial_{y_{i}} \Psi\right)_{y}\right] .
\end{aligned}
$$

It is easily seen that

$$
\begin{aligned}
& h^{-2} I \geqq \alpha\left\|Q_{h} \nabla_{y} u\right\|_{y}^{2}+\left(J_{2} a_{i}\left(T_{h} \nabla_{y} u\right), Q_{h} \partial_{y_{i}} u\right)_{y} \\
&-h^{-1}\left[\left(a_{i}\left(\nabla_{x} u\right),\left(e \cdot S_{h} \nabla_{x} u\right) \partial_{x_{i}} \Phi\right)_{x}+\left(a_{i}\left(\nabla_{y} u\right),\left(e \cdot T_{h} \nabla_{y} u\right) \partial_{y_{i}} \Psi\right)_{y}\right] .
\end{aligned}
$$

By writing $H=\left(h_{i \jmath}\right)$ in (4.6), we have 


$$
\begin{aligned}
\left(a_{i}\left(\nabla_{y} u\right),\left(\boldsymbol{e} \cdot T_{h} \nabla_{y} u\right) \partial_{y_{i}} \Psi\right)_{y}= & -\left(J a_{i}\left(S_{h} \nabla_{x} u\right),\left(\boldsymbol{e} \cdot \nabla_{x} u\right) \partial_{x_{i}} \Phi\right)_{x} \\
& -h\left(J a_{i}\left(S_{h} \nabla_{x} u\right),\left(\boldsymbol{e} \cdot \nabla_{x} u\right) \sum_{j} \partial_{x_{j}} \Phi \cdot h_{j i}\right)_{x}
\end{aligned}
$$

Hence

$$
\begin{aligned}
h^{-1}\left[\left(a_{i}\left(\nabla_{x} u\right),\left(e \cdot S_{h} \nabla_{x} u\right) \partial_{x_{i}} \Phi\right)_{x}+\left(a_{i}\left(\nabla_{y} u\right),\left(e \cdot T_{h} \nabla_{y} u\right) \partial_{y_{i}} \Psi\right)_{y}\right] \\
=\left(a_{i}\left(\nabla_{x} u\right),\left(e \cdot P_{h} \nabla_{x} u\right) \partial_{x_{i}} \Phi\right)_{x}+\left(h^{-1}\left(a_{i}\left(\nabla_{x} u\right)-a_{i}\left(S_{h} \nabla_{x} u\right)\right),\left(e \cdot \nabla_{x} u\right) \partial_{x_{i}} \Phi\right)_{x} \\
\quad-\left(J_{1} a_{i}\left(S_{h} \nabla_{x} u\right),\left(e \cdot \nabla_{x} u\right) \partial_{x_{i}} \Phi\right)_{x}-\left(J a_{i}\left(S_{h} \nabla_{x} u\right),\left(e \cdot \nabla_{x} u\right) \sum_{j} \partial_{x_{j}} \Phi \cdot h_{j_{i}}\right)_{x} .
\end{aligned}
$$

By our assumption and (5.4) it follows that

$$
h^{-2} I \geqq \alpha\left\|Q_{h} \nabla_{y} u\right\|_{y}^{2}-C\left(1+\|u\|_{1}\right)\left(\left\|Q_{h} \nabla_{y} u\right\|_{y}+\left\|P_{h} \nabla_{x} u\right\|_{x}+\|u\|_{1}\right) .
$$

Combining (5.2), (5.3) and this inequality, we proceed in parallel with the previous section. Then we can deduce the conclusion of Theorem 1 . Similarly Theorem 2 is proved for the solution $u$ of (5.1).

\section{REFERENCES}

[1] Beirão da Veiga, H., Equazioni ellittiche non lineari con ostacoli sottili, Ann. Scuola Norm. Sup. Pisa, 26 (1972), 533-561.

[2] Brezis, H. and Stampacchia, G., Sur la régularité de la solution d'inéquations elliptiques, Bull. Soc. Math. France, 96 (1968), 153-180.

[ 3 ] Caffarelli, G. V., Regolarità di un problema di disequazioni variazionali relativo a due membrance, Lincei Rend., Sci. Fis. Mat. Natur., 50 (1971), 659-662.

[4] Frehse. J., On the regularity of the solution of a second order variational inequality, Boll. Un. Mat. Ital., 6 (1972), 312-315.

[5] - On Signorini's problem and variational problems with thin obstacles, Ann. Scuola Norm. Sup. Pisa, 4 (1977), 343-362.

[6] Gerhardt, C., Regularity of solutions of nonlinear variational inequalities, Arch. Rational Mech. Anal., 52 (1973), 389-393.

[ 7 ] Grisvard, P., Régularité de la solution d'un problème aux limites unilatéral dans un domaine convexe, Séminaire Goulaouic-Schwartz (1975/76), Équations aux dérivées partielles et analyse fonctionelle, Exp. No.16, $11 \mathrm{pp.}$

[ 8 ] Hartman, P. and Stampacchia, On some nonlinear elliptic differential functional equations, Acta Math., 115 (1966), 271-310.

[9] Kinderlehrer, D. and Stampacchia, G., An introduction to variational inequalities and their applications, Academic Press, New York, 1980.

[10] Lewy, H., On a variational problem with inequalities on the boundary, J. Math. Mech., 17 (1968), 861-884.

[11] Lewy, H. and Stampacchia, G., On the regularity of the solution of a variational inequality, Comm. Pure Appl. Math., 22 (1969), 153-188.

[12] Lions, J. L., Quelques méthodes de résolution des problèmes aux limites non linéaires, Dunod Gauthier-Villars, 1969.

[13] —, Partial differential inequalities, Russian Math. Surveys, 27 (1972), 91-159.

[14] Marcus, M., The Dirichlet problem in domain whose boundary is partly degenerated, Ann. Mat. Pura Appl., 73 (1966), 159-194: 
[15] Williams, G. H., Lipschitz continuous solutions for nonlinear obstacle problems, Math. Z., 154 (1977), 51-65.

Kazuya Hayasida

Department of Mathematics

Faculty of Science

Kanazawa University

Kanazawa 920

Japan

Haruo Nagase

Suzuka College of Technology

Suzuka 510-02

Japan 